\title{
Studies on Genetic Variability, Correlation and Path Analysis in Yellow Pericarp Sorghum [Sorghum bicolor (L.) Moench] Genotypes
}

\author{
B. V. Vara Prasad* and V. Sridhar
}

Department of GPBR, College of agriculture, PJTSAU, Rajendranagar, Hyderabad-30, India

*Corresponding author

Keywords

Rainfall,

Temperature, Ranks

first, Sorghum,

Staple food, Rice,

Wheat and maize

Article Info

Accepted:

04 November 2019

Available Online:

10 December 2019

\section{A B S T R A C T}

The experiment was conducted under rainfed condition at Agricultural Research Station, Madhira during early rabi 2016 using 40 ICRISAT sorghum genotypes. Estimation of variation, phenotypic and genotypic correlations along with path analysis was calculated for the material under study. High amount of variability was recorded in the material for various traits under study. Plant height showed positive significant association with number of leaves per plant, leaf length, leaf width, ear length, straw weight, 100 seed weight and grain yield per plant. Number of leaves per plant showed positive significant association with leaf length, straw weight and number of leaves per plant. Path coefficient analysis revealed that plant height exhibited positive direct effect coupled with positive significant correlation with grain yield per plant. Plant height, number of leaves per plant and 100-seed weight are important characters in deciding the grain yield per plant in the present material under study.

\section{Introduction}

Sorghum, Sorghum bicolor (L.) Moench is an important staple food for more than 300 million people and feed for cattle in Asia and Africa. It is the fourth most important crop followed by rice, wheat and maize. India is a major sorghum growing country in the world, ranks first in acreage and second in production next to United States of America. In India sorghum is grown in areas receiving 500 to
$1000 \mathrm{~mm}$ annual rainfall with temperature ranging between 26 to $32^{\circ} \mathrm{C}$. It is grown in two seasons, viz., kharif as rainfed crop and rabi with protective irrigation thereby constituting 60 and 40 percent cultivation, respectively.

In India it is grown as a dual purpose crop serving both grain and fodder requirements of the farming community and its regional importance as a major food crop is as much as that of wheat and rice. 
The estimates of genetic variability indicated the amount of genetic variation present in the material which is necessary for sorghum breeding programmes. The correlation and path analysis in combination, can give a better insight, into cause and effect relationship between different pairs of characters. The correlation measures the relationship existing between pairs of traits. But dependent traits are an interaction product of many mutually associated components. The path analysis takes into account the cause and effect relationship between the variables by partitioning the association into direct and indirect effects through other independent variables. Grain yield is the product of interaction of component traits. Apart from correlation studies, path coefficient analysis is important to obtain information about different ways in which the component characters influences the grain yield.

\section{Materials and Methods}

The present experiment was conducted in deep black soil under rainfed condition at Agricultural Research Station, Madhira during early rabi 2016 using 40 yellow pericarp sorghum genotypes received from ICRISAT. The randomized block design was followed with three replications and each entry was sown in two rows of $3 \mathrm{~m}$ length with inter row spacing of $45 \mathrm{~cm}$ and intra row spacing of 15 $\mathrm{cm}$. All the recommended practices were followed to raise good crop. From each entry of every replication, five randomly selected plants were tagged for recording observations on all the quantitative characters. Days to 50 per cent flowering and days to maturity were recorded at plot level. Mean of five plants for each entry for each character was calculated and used for statistical analysis. Estimation of variation components and phenotypic and genotypic correlations were calculated by using the formulae given by Burton (1952) and Johnson et al., (1955). The simple correlation coefficient was subjected to path analysis (Dewey and Lu, 1959). The list of genotypes included in the experiment are depicted in Table 1.

\section{Results and Discussion}

Considerable amount of variability was observed in the material under study (Table 2). Plant height, leaf length, straw weight and grain yield showed highest values for heritability estimates indicating high degree of response to selection for these traits. Phenotypic and genotypic correlations were calculated for ten characters to know the nature of association existing among them. The results are presented in Table 3. Plant height showed positive significant association with number of leaves per plant, leaf length, leaf width, ear length, straw weight, 100 seed weight and grain yield per plant both at genotypic and phenotypic levels. Days to maturity showed positive significant association with ear length and straw weight.

Number of leaves per plant showed positive significant association with leaf length, straw weight and number of leaves per plant. This may be due to that the leaves contributed for higher photosynthates that resulted in more of dry matter production that enhanced straw weight and grain weight. Leaf length exhibited positive significant association with leaf width, ear length and 100 seed weight. Ear length and straw weight showed negative significant association with grain yield per plant. Selection of those traits showing positive significant association with grain yield should be done for yield gain in sorghum. These results are in agreement with the earlier findings of Umakanth et al., 2005, Patil et al., 2009, Prakash et al., (2010), Khandelwal et al., (2015) and Swamy et al., (2018). 
Table.1 List of yellow pericarp sorghum genotypes included in the study

\begin{tabular}{|c|c|c|c|c|c|c|c|}
\hline S.NO & Genotype & S.NO & Genotype & S.NO & Genotype & S.NO & Genotype \\
\hline $\mathbf{1}$ & IS -11163 & 11 & IS23053 & 21 & IS-18328 & 31 & IS-23457 \\
\hline $\mathbf{2}$ & IS -19479 & 12 & IS-9892 & 22 & IS-19106 & 32 & IS-14933 \\
\hline $\mathbf{3}$ & N-13 & 13 & IS-14950 & 23 & IS-22552 & 33 & IS-22415 \\
\hline $\mathbf{4}$ & IS-19001 & 14 & IS-22382 & 24 & IS-18460 & 34 & IS-24698 \\
\hline $\mathbf{5}$ & IS-2285 & 15 & IS-18398 & 25 & IS-19247 & 35 & IS-9868 \\
\hline $\mathbf{6}$ & IS-1134 & 16 & IS-18415 & 26 & IS-12034 & 36 & IS-10934 \\
\hline $\mathbf{7}$ & IS-9893 & 17 & IS-12503 & 27 & IS-3582 & 37 & IS-10306 \\
\hline $\mathbf{8}$ & N-14 & 18 & IS-2267 & 28 & IS-19238 & 38 & IS-22399 \\
\hline $\mathbf{9}$ & IS-19128 & 19 & IS-3508 & 29 & IS-18395 & 39 & IS-22895 \\
\hline $\mathbf{1 0}$ & IS-22479 & 20 & IS-19473 & 30 & IS-10918 & 40 & IS-18348 \\
\hline
\end{tabular}

Table.2 Variability estimates for ten traits in ICRISAT yellow pericarp sorghum genotypes

\begin{tabular}{|c|c|c|c|c|c|c|c|c|c|c|}
\hline & $\begin{array}{l}\text { Plant } \\
\text { height }\end{array}$ & $\begin{array}{c}\text { Days to } \\
50 \% \\
\text { flowering }\end{array}$ & $\begin{array}{l}\text { Days to } \\
\text { maturity }\end{array}$ & $\begin{array}{c}\text { No. of } \\
\text { leaves } \\
\text { per plant }\end{array}$ & $\begin{array}{c}\text { Leaf } \\
\text { length }\end{array}$ & $\begin{array}{c}\text { Leaf } \\
\text { width }\end{array}$ & $\begin{array}{c}\text { Ear } \\
\text { length }\end{array}$ & $\begin{array}{l}\text { Straw } \\
\text { weight }\end{array}$ & $\begin{array}{l}100 \text { seed } \\
\text { weight }\end{array}$ & $\begin{array}{c}\text { Grain } \\
\text { yield per } \\
\text { plant }\end{array}$ \\
\hline GCV & 37.849 & 6.671 & 2.296 & 22.980 & 17.279 & 8.810 & 31.294 & 78.478 & 32.171 & 32.153 \\
\hline PCV & 37.916 & 8.300 & 5.712 & 29.633 & 17.375 & 31.178 & 33.798 & 79.332 & 34.504 & 32.947 \\
\hline $\begin{array}{l}\mathbf{h}^{2}(\text { broad } \\
\text { sense) }\end{array}$ & 0.996 & 0.646 & 0.162 & 0.601 & 0.989 & 0.080 & 0.857 & 0.979 & 0.869 & 0.952 \\
\hline GA(5\%) & 206.613 & 8.214 & 1.995 & 3.970 & 24.698 & 0.359 & 11.822 & 983.534 & 15.501 & 2.093 \\
\hline GA(1\%) & 264.786 & 10.526 & 2.557 & 5.088 & 31.652 & 0.460 & 15.151 & 1260.452 & 19.866 & 2.682 \\
\hline $\begin{array}{c}\text { GA as } \% \text { of } \\
\text { mean } 5 \%\end{array}$ & 77.831 & 11.043 & 1.901 & 36.712 & 35.398 & 5.129 & 59.688 & 159.924 & 61.790 & 64.638 \\
\hline $\begin{array}{c}\text { GA as } \% \text { of } \\
\text { mean } 1 \%\end{array}$ & 99.745 & 14.153 & 2.436 & 47.048 & 45.364 & 6.573 & 76.494 & 204.952 & 79.187 & 82.837 \\
\hline
\end{tabular}


Table.3 Correlation coefficient estimates for ten characters in ICRISAT yellow pericarp sorghum genotypes

\begin{tabular}{|c|c|c|c|c|c|c|c|c|c|c|}
\hline & $\begin{array}{c}\text { Plant } \\
\text { height }\end{array}$ & $\begin{array}{c}\text { Days to } \\
\mathbf{5 0 \%} \\
\text { flowering }\end{array}$ & $\begin{array}{l}\text { Days to } \\
\text { maturity }\end{array}$ & $\begin{array}{c}\text { No. of } \\
\text { leaves per } \\
\text { plant }\end{array}$ & $\begin{array}{l}\text { Leaf } \\
\text { length }\end{array}$ & $\begin{array}{l}\text { Leaf } \\
\text { width }\end{array}$ & Ear length & $\begin{array}{l}\text { Straw } \\
\text { weight }\end{array}$ & $\begin{array}{c}100 \text { seed } \\
\text { weight }\end{array}$ & $\begin{array}{c}\text { Grain } \\
\text { yield per } \\
\text { plant }\end{array}$ \\
\hline Plant height & 1.0000 & $\begin{array}{c}0.1305 \\
(0.1427)\end{array}$ & $\begin{array}{c}0.0632 \\
(0.0712)\end{array}$ & $\begin{array}{c}0.7041 * * \\
(0.9267 * *)\end{array}$ & $\begin{array}{c}0.3816^{* * *} \\
(0.3849 * *)\end{array}$ & $\begin{array}{c}0.2085^{*} \\
\left(0.3039^{* *}\right)\end{array}$ & $\begin{array}{c}0.1944^{*} \\
\left(0.2038^{*}\right)\end{array}$ & $\begin{array}{c}0.3109 * \\
(0.3189 * *)\end{array}$ & $\begin{array}{c}0.2434 * * \\
(0.3563 * *)\end{array}$ & $\begin{array}{c}0.3081 * * \\
(0.3204 * *)\end{array}$ \\
\hline $\begin{array}{c}\text { Days to } 50 \% \\
\text { flowering }\end{array}$ & & 1.0000 & $\begin{array}{c}-0.0404 \\
(-0.0454)\end{array}$ & $\begin{array}{c}0.0729 \\
(0.1238)\end{array}$ & $\begin{array}{c}0.1090 \\
(0.1193)\end{array}$ & $\begin{array}{c}0.1824 * \\
\left(0.2990^{*}\right)\end{array}$ & $\begin{array}{c}0.1165 \\
(0.1345)\end{array}$ & $\begin{array}{c}0.2169 * \\
(0.2442 *)\end{array}$ & $\begin{array}{c}-0.0363 \\
(-0.0320)\end{array}$ & $\begin{array}{c}0.0432 \\
(0.0347)\end{array}$ \\
\hline $\begin{array}{l}\text { Days to } \\
\text { maturity }\end{array}$ & & & 1.0000 & $\begin{array}{c}0.0129 \\
(0.0551)\end{array}$ & $\begin{array}{c}-0.0708 \\
(-0.0785)\end{array}$ & $\begin{array}{c}-0.0453 \\
(-0.0655)\end{array}$ & $\begin{array}{c}0.2249^{*} \\
\left(0.2666^{* *}\right)\end{array}$ & $\begin{array}{c}0.2307 * \\
\left(0.2593^{*}\right)\end{array}$ & $\begin{array}{c}0.1674 \\
\left(0.2965^{*}\right)\end{array}$ & $\begin{array}{c}0.0914 \\
(0.1148)\end{array}$ \\
\hline $\begin{array}{c}\text { No. of leaves } \\
\text { per plant }\end{array}$ & & & & 1.0000 & $\begin{array}{c}0.3438 * * \\
(0.4577)\end{array}$ & $\begin{array}{c}0.1307 \\
(0.2937)\end{array}$ & $\begin{array}{c}0.1273 \\
(0.1866)\end{array}$ & $\begin{array}{c}0.3458 * * \\
(0.4496 * *)\end{array}$ & $\begin{array}{c}0.1696 \\
(0.4932 * *)\end{array}$ & $\begin{array}{c}0.2543 * * \\
(0.3648 * *)\end{array}$ \\
\hline Leaf length & & & & & 1.0000 & $\begin{array}{c}0.2769 * * \\
(0.3970)\end{array}$ & $\begin{array}{c}0.3729 * * \\
(0.3940 * *)\end{array}$ & $\begin{array}{c}-0.0359 \\
(-0.0337)\end{array}$ & $\begin{array}{c}0.2301 * \\
\left(0.3630^{* *}\right)\end{array}$ & $\begin{array}{c}0.0026 \\
(0.0106)\end{array}$ \\
\hline Leaf width & & & & & & 1.0000 & $\begin{array}{c}0.2768^{* *} \\
(0.3552 * *)\end{array}$ & $\begin{array}{c}0.2147^{*} \\
\left(0.3421^{* *}\right)\end{array}$ & $\begin{array}{c}0.2104^{*} \\
\left(0.5489^{* *}\right)\end{array}$ & $\begin{array}{c}0.0351 \\
(0.0539)\end{array}$ \\
\hline Ear length & & & & & & & 1.0000 & $\begin{array}{c}0.2608^{* *} \\
\left(0.2816^{* *}\right)\end{array}$ & $\begin{array}{c}0.1283 \\
\left(0.2526^{* *}\right)\end{array}$ & $\begin{array}{c}-0.3262 * * \\
(- \\
0.3529 * *)\end{array}$ \\
\hline Straw weight & & & & & & & & 1.0000 & $\begin{array}{c}0.1901^{*} \\
\left(0.3145^{* *}\right)\end{array}$ & $\begin{array}{c}-0.2063^{*} \\
(-0.2358 *)\end{array}$ \\
\hline $\begin{array}{c}100 \text { seed } \\
\text { weight }\end{array}$ & & & & & & & & & 1.0000 & $\begin{array}{c}0.1191 \\
\left(0.2010^{*}\right)\end{array}$ \\
\hline
\end{tabular}


Table.4 Path analysis estimates for ten traits in ICRISAT yellow pericarp sorghum genotypes

\begin{tabular}{|c|c|c|c|c|c|c|c|c|c|c|}
\hline & $\begin{array}{l}\text { Plant } \\
\text { height }\end{array}$ & $\begin{array}{c}\text { Days to } \\
50 \% \\
\text { flowering }\end{array}$ & $\begin{array}{l}\text { Days to } \\
\text { maturity }\end{array}$ & $\begin{array}{c}\text { No. of } \\
\text { leaves per } \\
\text { plant }\end{array}$ & $\begin{array}{c}\text { Leaf } \\
\text { length }\end{array}$ & $\begin{array}{c}\text { Leaf } \\
\text { width }\end{array}$ & $\begin{array}{c}\text { Ear } \\
\text { length }\end{array}$ & $\begin{array}{l}\text { Straw } \\
\text { weight }\end{array}$ & $\begin{array}{c}100 \text { seed } \\
\text { weight }\end{array}$ & $\begin{array}{c}\text { Grain } \\
\text { yield per } \\
\text { plant }\end{array}$ \\
\hline $\begin{array}{l}\text { Plant } \\
\text { height }\end{array}$ & $\begin{array}{c}0.3255 \\
(-1.4468)\end{array}$ & $\begin{array}{c}0.0425 \\
(-0.2064)\end{array}$ & $\begin{array}{c}0.0206 \\
(-0.1030)\end{array}$ & $\begin{array}{c}0.2292 \\
(-1.3408)\end{array}$ & $\begin{array}{c}0.1242 \\
(-0.5569)\end{array}$ & $\begin{array}{c}0.0679 \\
(-0.4397)\end{array}$ & $\begin{array}{c}0.0633 \\
(-0.2949)\end{array}$ & $\begin{array}{c}0.1012 \\
(-0.4615)\end{array}$ & $\begin{array}{c}0.0792 \\
(-0.5156)\end{array}$ & $\begin{array}{c}0.3081 * * \\
(0.3204 * *)\end{array}$ \\
\hline $\begin{array}{c}\text { Days to } \\
50 \% \\
\text { flowering }\end{array}$ & $\begin{array}{c}0.0158 \\
(0.0158)\end{array}$ & $\begin{array}{c}0.1211 \\
(0.1110)\end{array}$ & $\begin{array}{c}-0.0049 \\
(-0.0050)\end{array}$ & $\begin{array}{c}0.0088 \\
(0.0137)\end{array}$ & $\begin{array}{c}0.0132 \\
(0.0132)\end{array}$ & $\begin{array}{c}0.0221 \\
(0.03320\end{array}$ & $\begin{array}{c}0.0141 \\
(0.0149)\end{array}$ & $\begin{array}{c}0.0263 \\
(0.0271)\end{array}$ & $\begin{array}{c}-0.0044 \\
(-0.0036)\end{array}$ & $\begin{array}{c}0.0432 \\
(0.0347)\end{array}$ \\
\hline $\begin{array}{l}\text { Days to } \\
\text { maturity }\end{array}$ & $\begin{array}{c}0.0149 \\
(0.0416)\end{array}$ & $\begin{array}{c}-0.0095 \\
(-0.0265)\end{array}$ & $\begin{array}{c}0.2352 \\
(0.5844)\end{array}$ & $\begin{array}{c}0.0030 \\
(0.0322)\end{array}$ & $\begin{array}{c}-0.0167 \\
(-0.0459)\end{array}$ & $\begin{array}{c}-0.0107 \\
(-0.0383)\end{array}$ & $\begin{array}{c}0.0529 \\
(0.1558)\end{array}$ & $\begin{array}{c}0.0543 \\
(0.1515)\end{array}$ & $\begin{array}{c}0.0394 \\
(0.1732)\end{array}$ & $\begin{array}{c}0.0914 \\
(0.1148)\end{array}$ \\
\hline $\begin{array}{c}\text { No. of } \\
\text { leaves per } \\
\text { plant }\end{array}$ & $\begin{array}{c}0.1507 \\
(2.2786)\end{array}$ & $\begin{array}{c}0.0156 \\
(0.3045)\end{array}$ & $\begin{array}{c}0.0028 \\
(0.1354)\end{array}$ & $\begin{array}{c}0.2140 \\
(2.4588)\end{array}$ & $\begin{array}{c}0.0736 \\
(1.1254)\end{array}$ & $\begin{array}{c}0.0280 \\
(0.7221)\end{array}$ & $\begin{array}{c}0.0272 \\
(0.4589)\end{array}$ & $\begin{array}{c}0.0740 \\
(1.1055)\end{array}$ & $\begin{array}{c}0.0363 \\
(1.2127)\end{array}$ & $\begin{array}{c}0.2543 * * \\
(0.3648 * *)\end{array}$ \\
\hline Leaf length & $\begin{array}{c}-0.0478 \\
(-0.2222)\end{array}$ & $\begin{array}{c}-0.0137 \\
(-0.0689)\end{array}$ & $\begin{array}{c}0.0089 \\
(0.0453)\end{array}$ & $\begin{array}{c}-0.0431 \\
(-0.2642)\end{array}$ & $\begin{array}{c}-0.1253 \\
(-0.5772)\end{array}$ & $\begin{array}{c}-0.0347 \\
(-0.2292)\end{array}$ & $\begin{array}{c}-0.0467 \\
(-0.2274)\end{array}$ & $\begin{array}{c}0.0045 \\
(0.0195)\end{array}$ & $\begin{array}{c}-0.0288 \\
(-0.2095)\end{array}$ & $\begin{array}{c}0.0026 \\
(0.0106)\end{array}$ \\
\hline Leaf width & $\begin{array}{c}0.0284 \\
(0.2210)\end{array}$ & $\begin{array}{c}0.0249 \\
(0.2175)\end{array}$ & $\begin{array}{c}-0.0062 \\
(-0.0476)\end{array}$ & $\begin{array}{c}0.0178 \\
(0.2136)\end{array}$ & $\begin{array}{c}0.0377 \\
(0.2888)\end{array}$ & $\begin{array}{c}0.1363 \\
(\mathbf{0 . 7 2 7 4})\end{array}$ & $\begin{array}{c}0.0377 \\
(0.2584)\end{array}$ & $\begin{array}{c}0.0293 \\
(0.2488)\end{array}$ & $\begin{array}{c}0.0287 \\
(0.3993)\end{array}$ & $\begin{array}{c}\mathbf{0 . 0 3 5 1} \\
(\mathbf{0 . 0 5 3 9})\end{array}$ \\
\hline Ear length & $\begin{array}{c}-0.0736 \\
(-0.0607)\end{array}$ & $\begin{array}{c}-0.0441 \\
(0.0400)\end{array}$ & $\begin{array}{c}-0.0852 \\
(-0.0794)\end{array}$ & $\begin{array}{c}-0.0482 \\
(-0.0556)\end{array}$ & $\begin{array}{c}-0.1412 \\
(-0.1173)\end{array}$ & $\begin{array}{c}-0.1048 \\
(-0.1057)\end{array}$ & $\begin{array}{c}-0.3788 \\
(-0.2977)\end{array}$ & $\begin{array}{c}-0.0988 \\
(-0.0838)\end{array}$ & $\begin{array}{c}0.0486 \\
(-0.0752)\end{array}$ & $\begin{array}{c}-0.3262 * * \\
(- \\
0.3529 * *)\end{array}$ \\
\hline $\begin{array}{l}\text { Straw } \\
\text { weight }\end{array}$ & $\begin{array}{c}-0.1291 \\
(-0.3531)\end{array}$ & $\begin{array}{c}-0.0901 \\
(-0.2703)\end{array}$ & $\begin{array}{c}-0.0958 \\
(-0.2871)\end{array}$ & $\begin{array}{c}-0.1436 \\
(-0.4978)\end{array}$ & $\begin{array}{c}0.0149 \\
(0.0373)\end{array}$ & $\begin{array}{c}-0.0892 \\
(-0.3788)\end{array}$ & $\begin{array}{c}-0.1083 \\
(-0.3118)\end{array}$ & $\begin{array}{c}-0.4153 \\
(-1.1071)\end{array}$ & $\begin{array}{c}-0.0790 \\
(-0.3482)\end{array}$ & $\begin{array}{c}-0.2063 * \\
(-0.2358 *)\end{array}$ \\
\hline $\begin{array}{c}100 \text { seed } \\
\text { weight }\end{array}$ & $\begin{array}{c}0.0235 \\
(-0.1540)\end{array}$ & $\begin{array}{c}-0.0035 \\
(0.0138)\end{array}$ & $\begin{array}{c}0.0161 \\
(-0.1281)\end{array}$ & $\begin{array}{c}0.0163 \\
(-0.2131)\end{array}$ & $\begin{array}{c}0.0222 \\
(-0.1569)\end{array}$ & $\begin{array}{c}0.0203 \\
(-0.2372)\end{array}$ & $\begin{array}{c}0.0124 \\
(-0.1092)\end{array}$ & $\begin{array}{c}0.0183 \\
(-0.1359)\end{array}$ & $\begin{array}{c}0.0964 \\
(-0.4322)\end{array}$ & $\begin{array}{c}0.1191 \\
(0.2010 *)\end{array}$ \\
\hline
\end{tabular}


Fig.1 Genotypic path diagram of nine traits with grain yield per plant in ICRISAT yellow pericarp sorghum genotypes

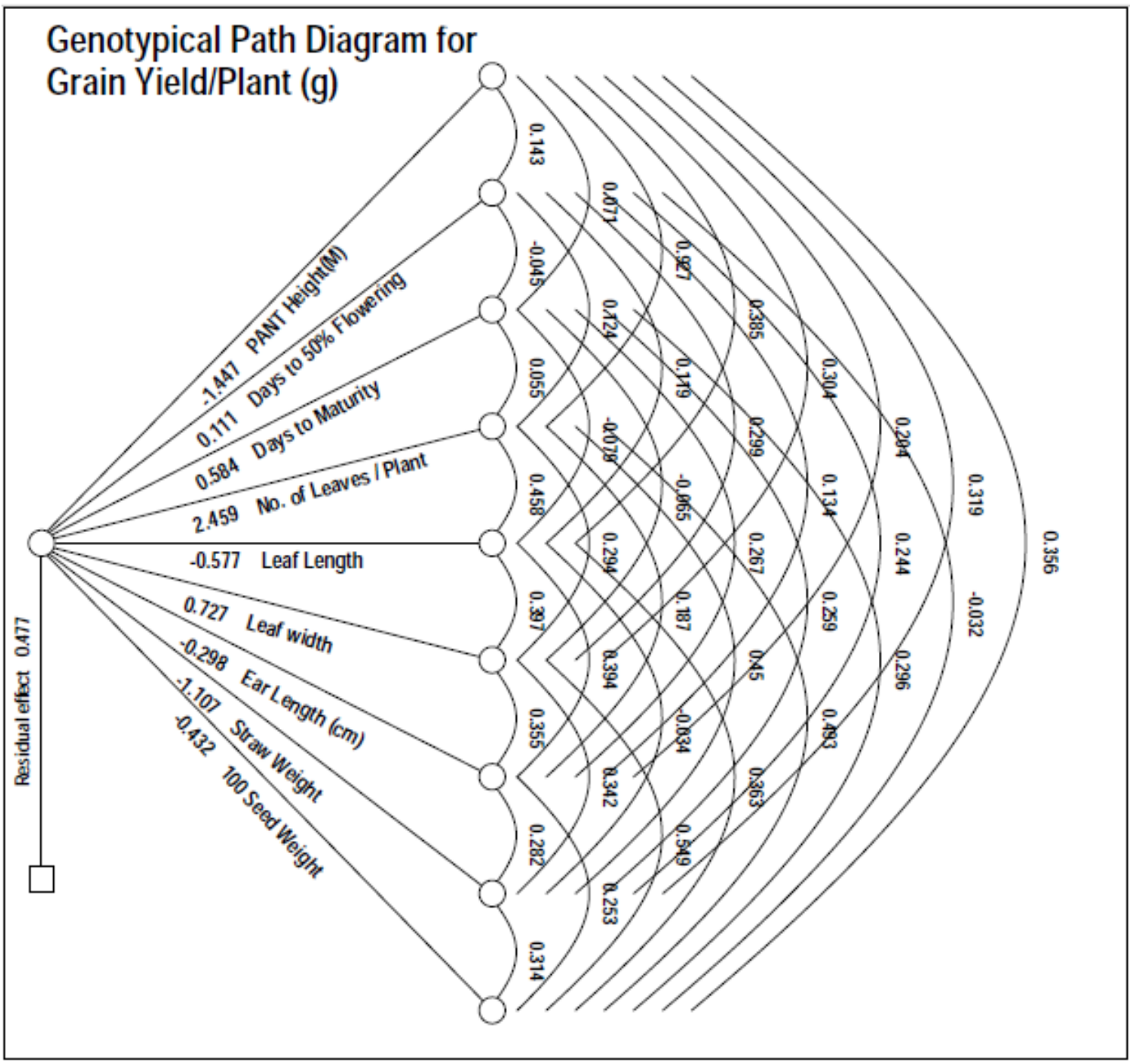

Path coefficient analysis (Table 4) revealed that plant height exhibited positive direct effect coupled with positive significant correlation with grain yield per plant (Fig. 1). Positive indirect effects were manifested through number of leaves per plant, leaf length and straw yield. Though, days to $50 \%$ flowering and days to maturity exhibited positive direct effects on grain yield, they did not show significant association with grain yield. Number of leaves per plant exhibited positive direct effect as well as positive significant correlation with grain yield per plant. Ear length and straw weight showed negative direct effect along with negative significant correlation with grain yield per plant. The negative indirect effects were manifested through all other traits included in the study. Similar results were reported by, Jain (2011), Jain and Patel (2013) and Soujanya et al., (2018). The results of path analysis indicated that plant height and number of leaves per plant showed highest positive direct effects along with positive significant correlation with grain yield in the present material under study. Thus, the present 
study indicated that the plant height, number of leaves per plant and 100-seed weight are important characters in deciding the grain yield per plant in the present material under study. Hence these characters may be considered as suitable selection indices in sorghum breeding programmes in the present investigation.

\section{References}

Burton, G.W. 1952. Quantitative inheritance in grasses. Proceedings of the 6th International Grassland Congress, pp. 227-283.

Dewey DR and Lu KH 1959. A correlation and path coefficient analysis of components of crested wheatgrass seed production. Agron. J. 51: 515- 518

Johnson H.W., Robinson H.F. and Comstock R.E. 1955. Genotypic and phenotypic correlations in soybean and their implications in selection. Agron. J., 47: 477-483.

Jain, S. K., 2011. Variation and association among fodder yield and other traits in germplasm of forage sorghum (Sorghum bicolor L. Moench). Indian Journal of Plant Genetic Resources., 24(3).

Jain, S.K. and Patel., P.R. 2013. Variability, correlation and path analysis studies in Sorghum [Sorghum Bicolor (L.) Moench] Forage Res., 39(1): Pp. 2730

Khandelwal V, Shukla M, Jodha BS, Nathawat VS, Dashora SK. Genetic parameters and character association in sorghum (Sorghum bicolor (L.) Moench). Indian Journal of Science and Technology, 2015; 8(22): 1-5. www.indjst. org

Patil, S.L., Sheelavantar, M.N. and Lamani, V.K. 2009. Correlation analysis among growth and yield components of winter sorghum. I $M$ N , 44: 14-17.

Prakash, R., Ganesamurthy, K., Nirmalakumari, A and Nagarajan, P. 2010. Correlation and path analysis in sorghum (Sorghum bicolor L. Moench). Electron J Plant Breed., 1(3): 315-318.

Soujanya, T, Shashikala, T and Umakanth, A.V. 2018. Correlation and path analysis in sweet sorghum [Sorghum bicolor (L.) Moench] hybrids for green fodder yield and its components. Bulletin of Environment, Pharmacology and Life Sciences, Vol 7(8): 52-56.

Swamy N, Biradar BD, Sajjanar GM, Ashwathama VH, Sajjan AS and Biradar AP, 2018. Genetic variability and correlation studies for productivity traits in Rabi sorghum [Sorghum bicolor (L.) Moench], Journal of Pharmacognosy and Phytochemistry; 7(6): 1785-1788.

Umakanth, A.V., Madhusudhana, R., Latha, K.M., Rafiq, S.M. and Kiran, V.S.S. 2005. Analysis of genetic variation and trait interrelationships in sorghum [Sorghum bicolor (L.) Moench]. National. J. Pl. Improv., 6(2): 104-107.

\section{How to cite this article:}

Vara Prasad, B. V. and Sridhar, V. 2019. Studies on Genetic Variability, Correlation and Path Analysis in Yellow Pericarp Sorghum [Sorghum bicolor (L.) Moench] Genotypes. Int.J.Curr.Microbiol.App.Sci. 8(12): 367-373. doi: https://doi.org/10.20546/ijcmas.2019.812.049 